Superalloys 2012: $12^{\text {th }}$ International Symposium on Superalloys

\title{
A CLOSED CONCEPT TO ASSOCIATE THE HOT-FORGING PROCESS CONTROLLED MICROSTRUCTURE WITH FATIGUE LIFE
}

\author{
Michael Stoschka ${ }^{1}$, Martin Stockinger ${ }^{2}$, Hermann Maderbacher $^{1}$, Martin Riedler ${ }^{2}$ \\ ${ }^{1}$ Chair of Mechanical Engineering, Montanuniversität Leoben; Franz-Josef-Str. 18; 8700 Leoben; Austria \\ ${ }^{2}$ Böhler Schmiedetechnik GmbH\&Co KG; Mariazellerstraße 25; 8605 Kapfenberg; Austria
}

Keywords: Fatigue analysis, Thermo-mechanical processing, Process simulation, Aerospace components.

\begin{abstract}
Description of a forging process which relates the fatigue life to microstructure needs to take into account several influencing factors throughout the entire hot-forging process. The calculation of dynamic fatigue strength based on static properties for hotforged components of superalloy 718, especially at elevated temperatures, is in general not possible.

The main objective of this paper is to present the capability of the 'Micro Structural Energy Approach' (MSEA), to determine the influence of different forging parameters on fatigue life, within a valid process window for aerospace components. This MSEA technique is based on extensive experimental data, and considers short-crack growth for damage assessment. The two parameters, viz. mean grain size and factor of heterogeneity, are used to link experimentally obtained life time results with its microstructure. These parameters are also capable of establishing a stable fatigue life prediction tool, by evaluation of the microstructure coupled with the hot-forging process simulation.
\end{abstract}

\section{Introduction}

In the process of finding a link between the microstructure and fatigue life, especially at elevated temperatures, numerous factors which influence the forging process must be taken into account. Static and dynamic recrystallisation, as well as distribution and shape of the delta-precipitates affect the endurable fatigue level [1]. The assessed microstructure can be evaluated by standard methods [2-4], leading to a uniform or bimodal grain distribution. The ASTM grain size $G$, as one-dimensional scalar value, is commonly used to assess the microstructure of the metallographic sections. In the case of profoundly textured parts, such as hot-forged components made of titanium alloy, a timeconsuming, cost-extensive three-dimensional microstructural assessment may be necessary to deduce manufacturing process dependent parameters. In this work, the characterization of the superalloy 718 grain distribution is carried out using the MSEAtechnique as first introduced in [5]. It enables a two-dimensional assessment of the microstructure, taking into account not only the grain distribution, but also considering the neighborhood of individual as-large-as grains.

A closed concept to generate a microstructural based evaluation method, linking the grain-shape based texture and morphology to fatigue life of hot-forged aerospace components made of superalloy 718, is depicted in Figure 1. The hot-forging process controlled microstructural formation and the local fatigue properties are connected as fatigue link by way of the MSEA parameters. The MSEA-approach was originally developed to afford an enhanced analysis of metallographic sections, taking the grade of bimodality into account. It is based on a two-parametric characterization of the microstructure and grain shape texture. Microstructural information in regard to grain morphology, statistical grain distribution, and as-large-as grains, are assessed simultaneously. The MSEA-approach provides two parameters for each evaluated metallographic section. Firstly, the mean grain size - expressed by the ASTM number $G$ or the microstructural energy parameter $e$ in terms of the MSEA-method - and secondly, the novel factor of heterogeneity $b$. Extensive experimental tests supported the construction of a microstructural dependent fatigue model using these two MSEA-parameters $e$ and $b$ for superalloy 718 [5]. Previous investigations have proven the statistical applicability of the MSEA-method and the sensitivity of the two parameters to identify the microstructure of hot-forged superalloy 718.

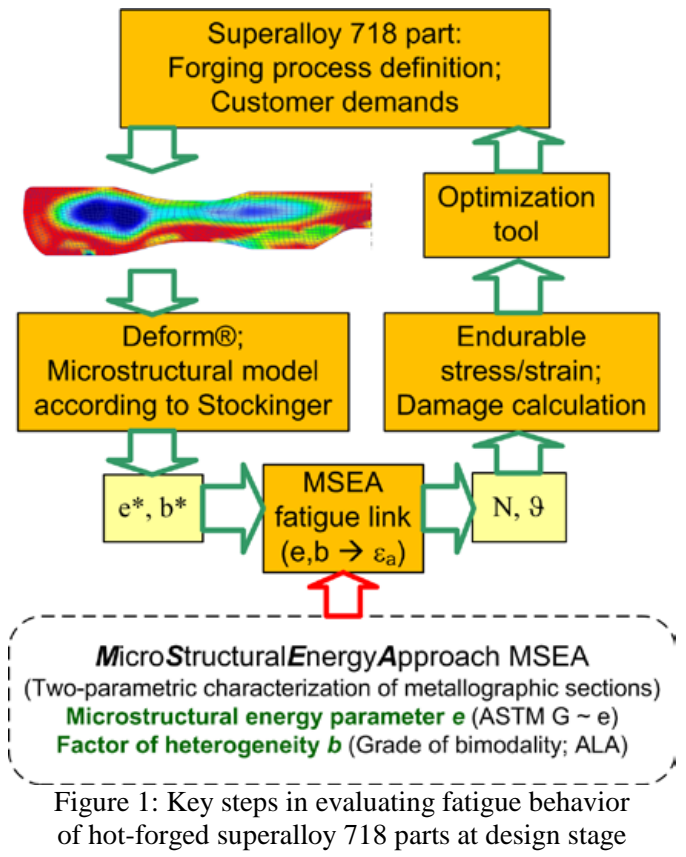

To close the gap to the numerical design stage, the two numerical parameters $e^{*}$ and $b^{*}$ were derived by the use of advanced hotforging simulation tools [6]. The two MSEA-parameters $e$ and $b$ establish a closed design loop to connect the local fatigue behavior and the local hot-forging process properties for superalloy 718 parts. 


\section{Survey of Microstructural Characterization}

The 'Micro Structural Energy Approach' benchmarks the microstructure in an alternative way supporting a two-parametric characterization technique as follows. The microstructural fatigue life mainly depends on the mode and occurrence of crack growth channels on slip bands. The fast single slip crack growth or the slower double slip crack growth can occur inside the grain, depending on the crystallographic orientation and dislocation appearance [7]. Once the crack tip reaches a boundary, a comparatively high energy input is needed to overcome this barrier. Owing to this crack growth, a plane description of shape and contiguity of the individual grains is used as fracture mechanical background.

The first output of the MSEA, the microstructural energy parameter $e$, is linked to the average ASTM grain size $G$. On the other hand, the second output, the factor of heterogeneity $b$, is a numerical representation of the degree of bimodality, focusing on coarse grains taking the adjacency matrix into account. Figure 2 displays the relation between standard ASTM grain size $G$ and the microstructural energy $e$, which in principal, corresponds to the reciprocal grain diameter. The relation between $G$ and $e$ can be derived as exponential function [6].

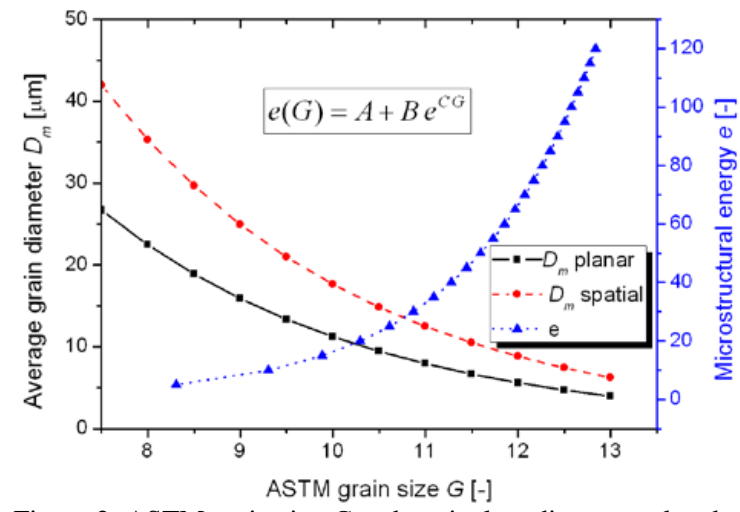

Figure 2: ASTM grain size $G$ and equivalent diameter related to the 'synthetic' microstructural energy $e$

The microstructural energy parameter $e$ varies between two and one-hundred forty-five, whereas the corresponding grain size number $\mathrm{G}$ lies in between seven and fourteen. The microstructural parameter $e$ is inversely proportional to the equivalent circle diameter; i.e., a microstructural energy value of one-hundred and twenty leads to a grain diameter of about four microns. The MSEA-parameter $e$ is sensitive to fine-grained microstructures and is therefore most suitable for hot-forging applications. For easier customer identification, the ASTM grain number $G$ or the equivalent diameter $D_{m}$ can be used instead.

The relation between both MSEA-parameters $e$ and $b$ and the underlying microstructure is illustrated in Figure 3. The images exhibit a mean ASTM grain size of $G=11.5$ but show distinct differences in individual grain size, distribution, and adjacency matrix. This leads to a comparably huge variation in the heterogeneity. The higher the factor of heterogeneity, the more bimodal is the evaluated microstructure. The innovative factor of heterogeneity $b$ describes the amount and connectivity of as-largeas grains. This factor can therefore not be easily replaced by the standard deviation of the evaluated particle-based grain distribution.
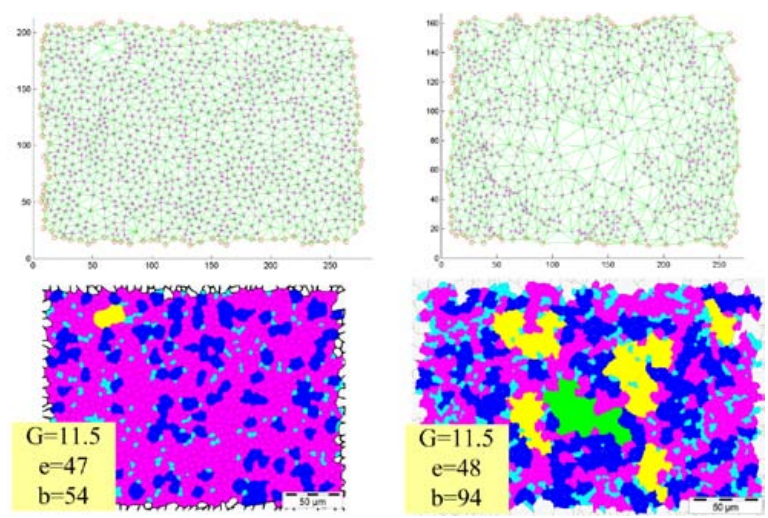

Figure 3: Triangulated MSEA-beam elements and particle based grain size information of two metallographic sections

To characterize the microstructure of metallographic sections using the automated microstructural energy approach, two major steps are necessary.

Firstly, a particle analysis must be accomplished. The principal output of this step is a table containing geometric shape properties of each detected particle. Only a few independent geometric grain properties are used for further processing [8]. The linear independent grain properties were identified by principalcomponent-analysis. The human-machine-interface covering the particle-based grain analysis, as well as an evaluated MSEAresult, is displayed in Figure 4. The application is implemented in the software package AnalySiS ${ }^{\circledR}$ as user-defined modules. AnalySiS provides the tools for grain detection, and for assessment of the individual grain properties. The photo taken from the metallographic section must be cleaned from twins, carbides, and huge delta-phase particles, to facilitate grain boundary detection.

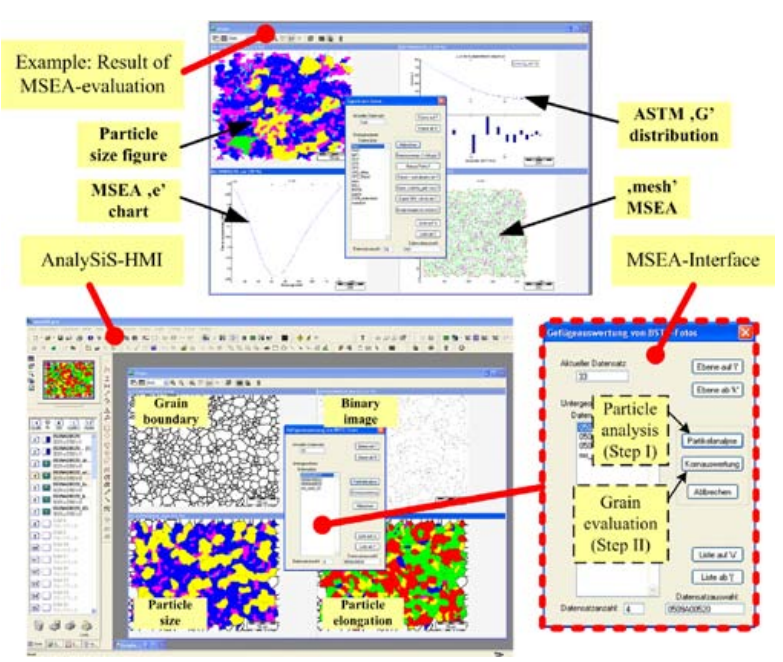

Figure 4: Interface of the MSEA-tool for automated characterization of metallographic sections

Secondly, the evaluated planar grain distribution is automatically transferred into a triangulated beam-element mesh using equivalent geometric properties as local stiffness parameters. Finally, load is applied uniformly on this synthetic matrix, which 
leads to a characteristic distribution of the evaluated synthetic distortion energy. The statistical interpretation of this energy curve leads to the two MSEA-parameters: microstructural energy $e$ - as mean value - and factor of heterogeneity $b$ as degree of inequality of the investigated grain distribution. The implemented core of the MSEA-module consists of compiled user-defined Matlab ${ }^{\circledR}$-procedures. These executables cover meshing of the synthetic microstructure, call of the linear-elastic finite element solver, and the subsequent post-processing routines to determine the MSEA-parameters.

Both MSEA-parameters 'synthetic' microstructural energy $e$ and factor of heterogeneity $b$ define further on the parametric link between hot-forging process and dynamic test results of superalloy 718 .

\section{Fatigue Assessment}

Assessment of the fatigue behavior can be done using stress- or strain-based approaches. At the time of conception of the MSEAmethod, most of the dynamic tests were done at room temperature and under stress control [5]. During the subsequent stages of improvement in the development cycle, results from fatigue tests of specimen cut-up from aerospace components were included [6]. These strain-controlled, low-cycle-fatigue tests covered both ambient and elevated temperatures up to $650^{\circ} \mathrm{C}$. The description of the $\varepsilon$-N-curves was done in accordance to the four-parametric Mansin-Coffin-law. Equation 1 denotes the total strain amplitude $\varepsilon_{a}$ which consists of elastic $\varepsilon_{a . e l}$ and plastic part $\varepsilon_{a, p l}$.

$\varepsilon_{a}=\varepsilon_{a . e l}+\varepsilon_{a . p l}=\frac{\sigma_{f}{ }^{\prime}}{E}(2 N)^{b}+\varepsilon_{f}{ }^{\prime}(2 N)^{c}$

The elastic strain amplitude depends on the fatigue coefficient $\sigma_{f}$, the young modulus $E$, and the fatigue exponent $b$. The plastic strain is determined by the ductility coefficient $\varepsilon_{f}$ ' and the cyclic ductility exponent $c$. In the case of hot-forging process, it has to be kept in mind that each parameter is influenced by the grain size and the temperature. Further on, gamma prime precipitations and carbides lead to a significant precipitation strengthening at elevated temperatures [1].

At this stage, a neural network consisting of two-layers is used to assess the $\varepsilon$-N-relationship. The corresponding layout is shown in Figure 5. The network was trained with strain-controlled fatigue test results covering a temperature range from $20^{\circ} \mathrm{C}$ to $650^{\circ} \mathrm{C}$. The stress based test values were converted into the strain range taking both the mean stress ratio and the stress gradient into account [6].

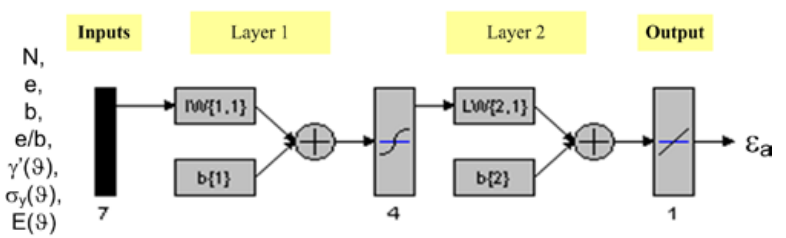

Figure 5: Neural network layout used to assess the $\varepsilon$-N-relation

The whole data was divided into three groups; randomly chosen three-hundred eighty points to train the network, one-hundred and twenty points to test it, and finally, one-hundred points for simulation to validate the feed-forward neural network. The first implemented layer is sigmoid and the second layer uses a linear function. Input parameters of the neural network are:

- number of endurable load cycles $N$,

- mean grain size - in term of the MSEA-value $e$,

- factor of heterogeneity $b$,

- ratio of $e$ to $b$ to indicate the spread of the grain distribution,

- $\quad$ precipitation hardening $\gamma^{\prime}(\vartheta)$,

- $\quad$ yield strength $\sigma_{y}(\vartheta)$ and finally

- $\quad$ young modulus $E(\vartheta)$.

The influence of operating temperature is already covered within the notified parameters. Output from the network is the strain amplitude $\varepsilon_{a}$ at the requested temperature level and number of load cycles, which is also influenced by the local microstructural properties due to the hot-forging process.

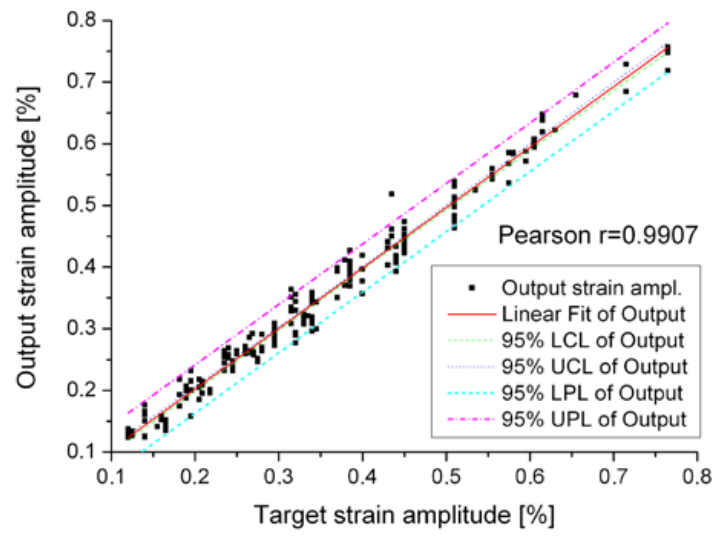

Figure 6: Regression between output and target strain amplitude of the neural network predicting the $\varepsilon$-N-behavior

The neural network offers satisfying correlation behavior within its data point limits. Figure 6 shows the achieved regression between the target values and the output strain amplitude. A twotailed test leads to the Pearson regression value of 0.991 . The 95\%-confidence interval for the linear fit is also sketched into the diagram. Additionally, the 95\%-prediction range covering the data points is plotted.

\section{Simulation Chain}

The hot-forging process can already be simulated at design stage using advanced simulation tools. The abbreviated flowchart is shown in Figure 1. The extended simulation chain, split up into several major tasks, is shown in Figure 7. It is structured into different modules which perform user-specific tasks.

The metal forming simulation tool Deform ${ }^{\circledR}$ defines the basic software. Substantial extensions with regard to grain growth evolution were added as Fortran ${ }^{\circledR}$-subroutines to implement the microstructural model introduced by Stockinger [5]. The output parameters of the microstructural energy approach, $e$ and $b$, are used as linking values to achieve a closed simulation chain. 


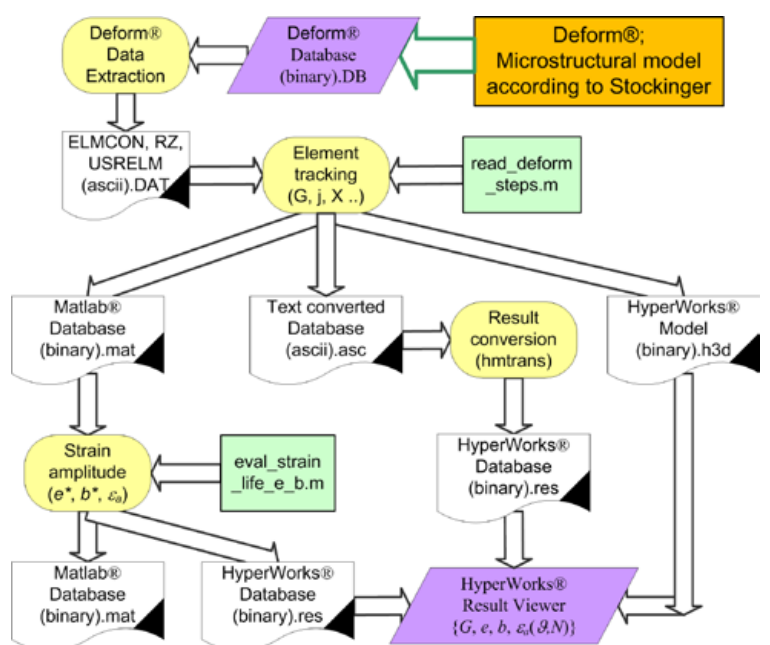

Figure 7: Extended flowchart of simulation chain between the interacting program modules

The mean ASTM grain size is directly available as output variable by the implemented user-defined microstructural model [9], and only needs slight accommodation to fit the process chain thoroughly. This can be explained by the fact that the simulated ASTM grain size represents the mean value and not the scatter band within the grains. The achieved value is named $e^{*}$. This adaptation was first introduced in [6].

$$
e^{*}\left(G_{\text {avg }}, X(t), \varphi(t)\right)=\frac{e\left(G_{a v g}\right)}{f_{1}(X(t), \varphi(t))}
$$

Effort has to be taken to derive the factor of heterogeneity $b^{*}$ from the microstructural simulation process parameters. Local recrystallization behavior influences the occurrence of coarse grain structures and hence, has to be taken into consideration.

$b^{*}(\Delta D(t), X(t), \varphi(t))=C_{3} \frac{f_{1}(X(t), \varphi(t))}{f_{2}(\Delta D(t))}$

In addition, the simulation-step-based growth in local grain size also influences the bimodality. Equations 2 and 3 reveal the implemented equations [6]. Although the current simulation model is adjusted to the experimental test results at customerdefined points, it is scheduled to incorporate constitutive models also, especially in regard to the factor of heterogeneity.

Forging process simulation includes, owing to the large displacements, a couple of re-meshing steps. This is somewhat challenging when the time-dependent distribution of numerous properties is necessary outside the hosting simulation program. To support the availability of the element-based forging results, including change in microstructure even outside Deform ${ }^{\circledR}$, data extraction of the element-based results into text files was done first. These files were analyzed by user-defined Matlab ${ }^{\circledR}$-routines, which convert the whole numerical forging process results into binary databases. To translate the ASCII-results to the binary result format, specific Altair-Hyperwork ${ }^{\circledR}$ C-libraries were used. This enables the use of Altair-HyperView ${ }^{\circledR}$ player to inspect the results or include them into presentation slides for customer visualization. Due to the fact that the ASCII export within Deform is based on elemental properties, only one integration point covers the elemental results. This coarsens the time-dependent elemental results a bit, but the general behavior is unchanged. Figure 8 shows the change in the simulated grain diameter $D_{m}$ of a hotforged turbine disc at three characteristic points.

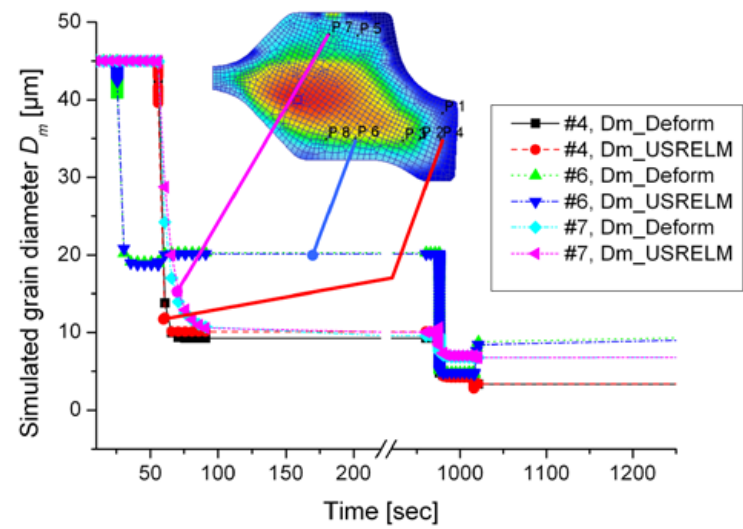

Figure 8: Point tracking of simulated average diameter comparing Deform ${ }^{\circledR}$ to the reduced elemental results

The hot-forging process only covers fractions of a second and is therefore identified by a lot of simulation steps within a very small time range. Each line marker in Figure 8 represents such a step. It has to be stated that the size of the elements has an influence when tracking the elemental results back to the start of the forging process. Depending on the re-meshing results during the forging simulation, it is possible to switch to an adjacent element possessing a different time-dependent microstructural history.

The contour plots with and without averaging in Figure 9 showed that adjacent elements possessed an incremental grading without any transient effects due to the data translation. Exemplarily pictured is the grain diameter $D_{m}$ of a turbine disc immediately after pre-forming ( 100 $s$ in Figure 8), after reheating or rather before final pressing $(\sim 950 \mathrm{~s})$, and after final cooldown ( 3600 s).
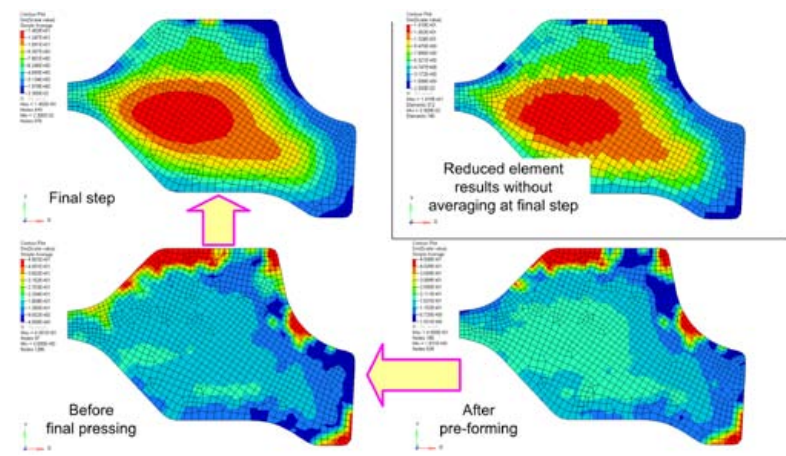

Figure 9: Predicted evolution of average grain diameter at characteristic time steps during hot-forging of superalloy 718

Figure 10 illustrates the ASTM grain size as characteristic simulation result, and the total strain, for three hot-forged turbine 
discs using the microstructural model according to Stockinger [5]. These characteristic parts are further on used for a case study reflecting the strain-controlled fatigue life versus parameter settings of the hot-forging processes.
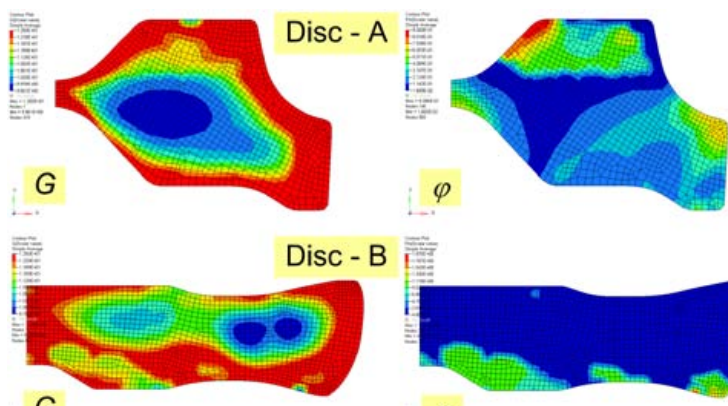

G
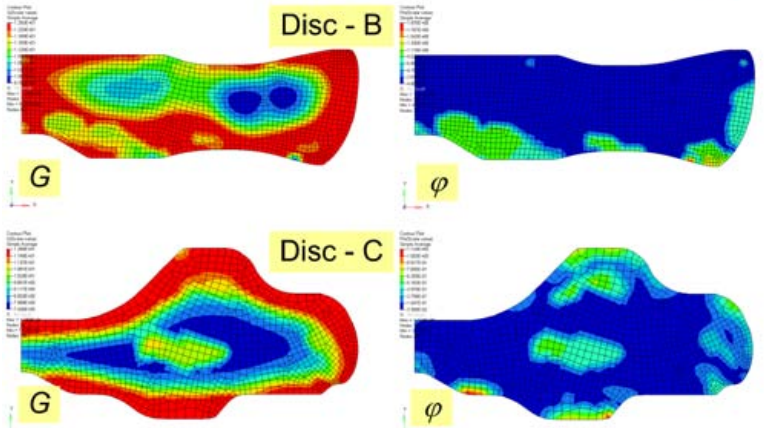

$\varphi$

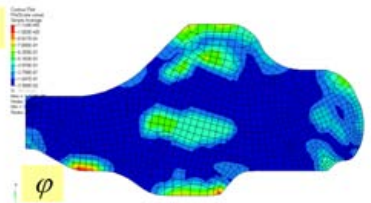

Figure 10: Examples of ASTM grain size (left) and total strain (right) distribution of three characteristic turbine discs

As displayed in Figure 7, the ASCII data export not only enables the use of customer dependent post-processing interfaces, but is also necessary to assess the lifetime of the hot-forged component. This is done by applying the introduced neural network onto the elemental and time-dependent microstructural simulation results. The implementation was again done as Matlab ${ }^{\circledR}$-code. The assessed strain amplitude depends on operating temperature and number of endurable load cycles. Here, the adapted MSEAparameters $e^{*}$ and $b^{*}$ are used as interface values. Figure 11 depicts the corresponding contour plots for the three investigated discs.

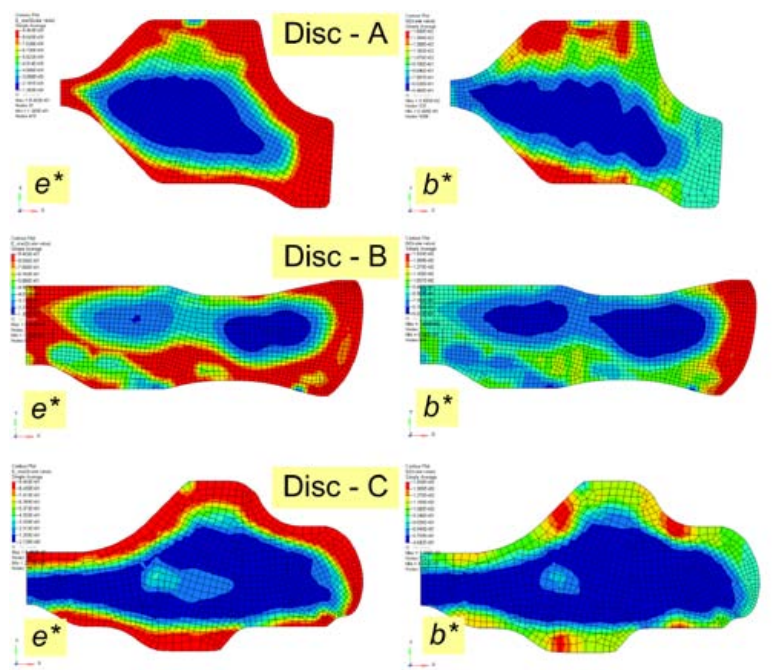

Figure 11: Enhanced microstructural simulation tool results of the three sample turbine discs
The mapping between ASTM grain size and microstructure energy is clearly recognizable. The distribution of the bimodality of the microstructure - assessed by the factor of heterogeneity - is shown in the bottom row subfigures in Figure 11.

The regression behavior of assessing the strain amplitude of hotforged superalloy 718 components by simulation is shown for the three sample discs in Figure 12. The specimens were taken from turbine disc cut-ups and dynamically tested under stabilized strain control. The investigated tests range from $20^{\circ} \mathrm{C}$ up to $650{ }^{\circ} \mathrm{C}$.

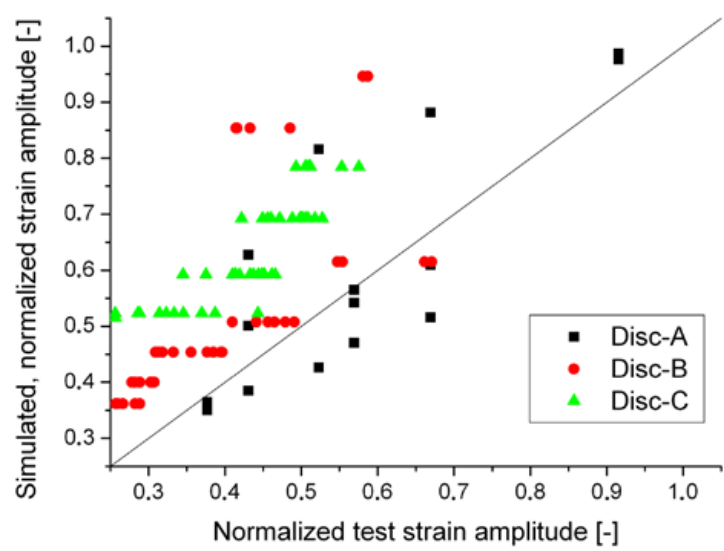

Figure 12: Examples of ASTM grain size and total strain distribution of three characteristic turbine discs

In addition, the test samples partially exhibited a relatively wide scatter band at selected levels. The $\varepsilon$ - $\mathrm{N}$-neural network was fed with the seven input values depicted in Figure 7. Although this $\varepsilon$ $\mathrm{N}$-assessment seems to be non-conservative, it can be used for comparison purposes. It is programmed to test strain-controlled specimens at elevated temperature levels at the test laboratory to narrow the scatter band further on.
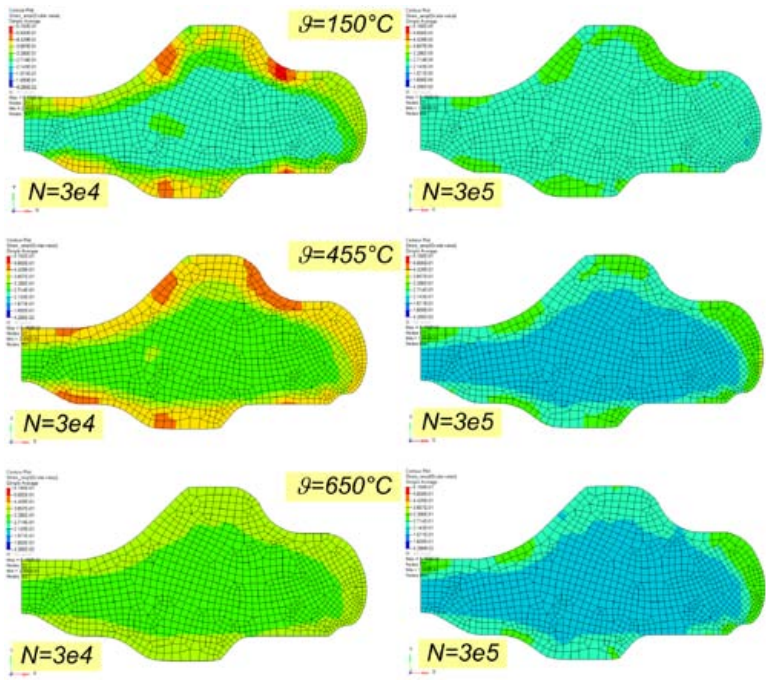

Figure 13: Proposed strain by the closed simulation chain for elevated temperature in the LCF and HCF-region (disc-C) 
The simulated strain amplitude is related both to the mean grain size and the local bimodality. Figure 13 depicts the corresponding simulated fatigue results for sample disc-C at temperature levels of $150^{\circ} \mathrm{C}, 455^{\circ} \mathrm{C}$ and $650^{\circ} \mathrm{C}$, and compares the endurable strain amplitude at two different load cycles. The legend threshold is fixed to visualize the local change in fatigue at specific operating conditions.

\section{Case Study}

The simulation chain is closed for hot-forged aerospace components made of superalloy 718 . As a significant advantage, this method enables a fatigue sensitivity study of changes in the hot-forging process at design stage itself.

To determine the influence of changes of hot-forging process parameters on a turbine disc, a sensitivity study was done in [11]. Investigated parameters of the hot-forging process were the initial billet temperature, die temperatures, environment temperature during forging process, furnace temperature during heat treatment, friction between dies and work piece, heat transfer between dies and work piece, die speed, resting time, reheating and transfer time for a specific disc.

The sensitivity study with regard to mean grain size brought out the main parameters for the investigated hot-forging process of a turbine disc made of superalloy 718 as follows.

- $\quad$ Free resting time before final pressing

- $\quad$ Pressing after pre-forming transfer

- Billet temperature

- $\quad$ First pre-forming step

- $\quad$ Second pre-forming step

- Final pressing

These key parameters were modified within the recommended hot-forging process limits given in [11]. The subsequent change in endurable strain range is plotted in Figure 14. Investigations showed that the changes are not throughout the part, but concentrated on local regions.

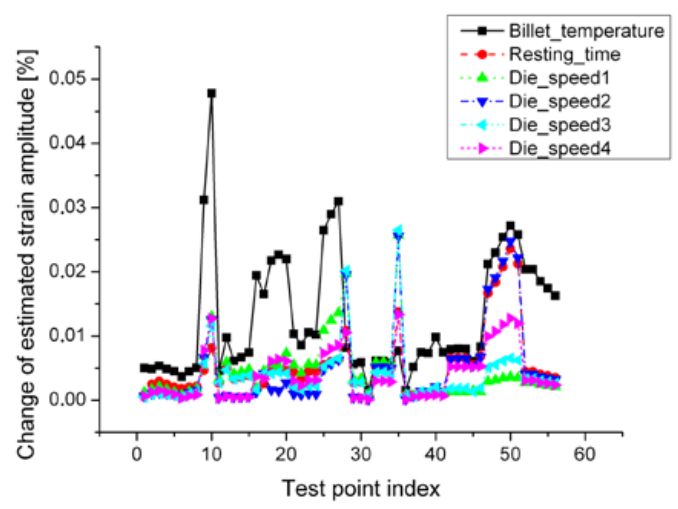

Figure 14: Sensitivity study of hot-forging parameters by simulation; exemplified for sample disc-C

The observed variations in fatigue life are comparably small. But this statement is only valid as long as the investigated hot-forging parameters are within the process window of the grain size. For instance, local overheating during the forging step dissolves the delta-phase and finally leads to coarse grains which reduce the fatigue level. This assumption is confirmed by the identification of billet temperature as the most important process parameter.

The specified fatigue life results were evaluated at characteristic test points and also at specific temperature levels. Indices number ten, fifteen, twenty-eight and fifty in Figure 14 highlight the anticipated local influence of hot-forging process parameters on fatigue life.

\section{Conclusion}

The microstructural energy approach supports an alternative description of the microstructure. The microstructural energy parameter $e$ correlates to the ASTM grain size $G$. The factor of heterogeneity $b$ characterizes the amount of both non-equiaxed and large-sized grains in a unique manner without additional analysis work. These microstructural values support the establishment of a closed-loop chain to assess the endurable strain amplitude of hot-forged components at design stage itself.

The temperature and microstructural dependency of the $\varepsilon-\mathrm{N}$ curves was assessed by a two-layer neural network. A closed loop model capable to estimate the strain-controlled fatigue life was introduced. Its applicability on three turbine disc samples was illustrated.

Investigations showed that the mean ASTM grain size dominates the fatigue life characteristic, but the local fatigue properties are nevertheless influenced by the heterogeneity and time-dependent evolution of the microstructure. Depending on the location of the assessed point the priority of the process parameters changes. Nevertheless, the billet temperature stays at the top in this regard. The entire hot-forging development process, including an integrated assessment of local strain-based fatigue behavior, has been illustrated for superalloy 718 turbine disc applications.

\section{Acknowledgements}

The Austrian Federal Government and the Styrian Provincial Government, represented by the Styrian promotion of economic development, within the research activities of the K2 Competence Centre on "Integrated Research in Materials, Processing and Product Engineering", operated by the Materials Center Leoben Forschung $\mathrm{GmbH}$ under the frame of the Austrian COMET Competence Centre Programme, is gratefully acknowledged.

\section{References}

1. Roger C. Reed, The Superalloys Fundamentals and Applications (New York, NY: Cambridge University Press, 2008), 74-89.

2. ASTM Standard E 112, 1996e2, "Standard Test Methods for Determining Average Grain Size”, ASTM International, West Conshohocken, PA, www.astm.org.

3. ASTM Standard E 1181, 2002, "Standard Test Methods for Characterizing Duplex Grain Sizes”, ASTM International, West Conshohocken, PA, www.astm.org.

4. ASTM Standard E 930, 1999, "Standard Test Methods for Estimating the Largest Grain Observed in a Metallographic Section (ALA Grain Size)”, ASTM International, West Conshohocken, PA, www.astm.org. 
5. M. Stoschka et al., "Assessment of Lifetime Calculation of Forged IN718 Aerospace Components Based on a MultiParametric Microstructural Evaluation”, Superalloys, 2008, 573582.

6. M. Stoschka et al., "An Integrated Approach to relate Hot Forging Process Controlled Microstructure of IN718 Aerospace Components to Fatigue Life", Proceedings of the $7^{\text {th }}$ International Symposium on Superalloy 718 and Derivatives, (2010), 751-765.

7. O. Düber et al., "Experimental characterization and two dimensional simulation of short-crack propagation in an austenitic-ferritic duplex steel”, International Journal of Fatigue, 28 (2006), 983-992.

8. H. Maderbacher et al. "Link from the Microstructure to the Fatigue Lifetime of Forged Inconel 718 Components”, DanubiaAdria Symposium on Advances in Experimental Mechanics, 26 (2009), 59-60.

9. M. Stockinger, and J. Tockner, "Optimizing the Forging of Critical Aircraft Parts by the Use of Finite Element Coupled Microstructure Modeling", Proceedings of the $6^{\text {th }}$ International Special Emphasis Symposium on Superalloys 718, 625, 706 and Derivatives, (2005), 517-526.

10. M. Stockinger et al., "Modeling of DELTA-Phase Dissolution During Preheating of INCONEL718 Turbine Disks", Proceedings of the $5^{\text {th }}$ International Special Emphasis Symposium on Superalloys 718, 625, 706 and Derivatives, (2001), 141-148.

11. H. Maderbacher et al., "Lifetime optimization of hot forged aerospace components by linking microstructural evolution and fatigue behaviour”, Advanced Materials Research, 278 (2011), 162-167. 